\title{
Increased expression and secretion of recombinant hIFNy through amino acid starvation-induced selective pressure on the adjacent HIS4 gene in Pichia pastoris
}

Original research article/Review

Ali Razaghi ${ }^{13,4}$, Roger Huerlimann ${ }^{1,4}$, Leigh Owens ${ }^{2}$, Kirsten Heimann 1,3,4,区

\author{
${ }^{1}$ College of Marine and Environmental Sciences, James Cook \\ University, Townsville 4811, Queensland, Australia \\ ${ }^{2}$ College of Public Health, Medical and Veterinary Sciences, \\ James Cook University, Townsville, QLD, 4811, Australia \\ ${ }^{3}$ Centre for Biodiscovery and Molecular \\ Development of Therapeutics, James Cook University, \\ Townsville4811, Queensland, Australia \\ ${ }^{4}$ Comparative Genomics Centre, James Cook University, \\ Townsville 4811, Queensland, Australia
}

Received August 10, 2015, accepted October 31, 2015

\begin{abstract}
Transcriptional co-regulation of adjacent genes has been observed for prokaryotic and eukaryotic organisms, alike. High levels of gene adjacency were also found in a wide variety of yeast species with a high frequency of co-regulated gene sets. The aim of this research was to study how selective pressure on the Histidinol dehydrogenase gene (HIS4), using amino acid starvation, affects the level of expression and secretion of the adjacent human interferon gamma gene ( $h I F N y$ ) in the recombinant Pichia pastoris GS115 strain, a histidine-deficient mutant. hIFN $\gamma$ was cloned into the $p$ PIC9 vector adjacent to the HIS4 gene, a gene essential for histidine biosynthesis, which was then transformed into $P$. pastoris. The transformed $P$. pastoris was cultured under continuous amino acid starvation in amino acid-free minimal medium for ten days, with five inoculations into unspent medium every second day. Under these conditions, only successfully transformed cells ( $\left.h I F N \gamma-H I S 4^{+}\right)$are able to synthesise histidine and therefore thrive. As shown by ELISA, amino acid starvation-induced selective pressure on HIS4 improved expression and secretion of the adjacent $h I F N \gamma$ by $55 \%$ compared to unchallenged cells. RT-qPCR showed that there was also a positive correlation between duration of amino acid starvation and increased levels of the hIFNY RNA transcripts. According to these results, it is suggested that these adjacent genes ( $h I F N y$ and HIS4) in the transformed P. pastoris are transcriptionally co-regulated and their expression is synchronised. To the best of the knowledge of the authors; this is the first study demonstrating that amino acid starvationinduced selective pressure on HIS4 can alter the regulation pattern of adjacent genes in P. pastoris.
\end{abstract}

Keywords Human interferon gamma-Histidinol dehydrogenase-Gcn4p-Serial passage-Transcriptional co-regulation

\section{INTRODUCTION}

There is increasing evidence that eukaryotic genes are co-regulated based on their location within the genome. Adjacent genes are subjected to tighter transcriptional coregulation compared to distantly placed genes. This type of co-regulation appears to be an evolutionary conserved and a vital regulatory mechanism in eukaryotes including yeasts and has a functional significance for maintaining coordinated levels of gene expression (Arnone et al., 2012). For example, adjacent genes in Saccharomyces cerevisiae display similar patterns of expression (Kruglyak \& Tang, 2000), which is substantiated by genome-wide expression studies in a number of organisms, such as Drosophila (Boutanaev et al., 2002), nematodes (Lercher et al., 2003), mice (Purmann et al., 2007), humans (Purmann et al., 2007), and Arabidopsis (Arnone et al., 2012; Wei et al., 2011; Williams \& Bowles, 2004).
Another remarkable example are the genes encoding ribosomal proteins and the rRNA biosynthesis pathway exhibiting a high percentage of adjacent gene pairs (Wade et al., 2006). This phenomenon is wide-spread in a variety of yeast species with approximately $24 \%$ of the ribosome and rRNA biosynthesis genes being positioned as adjacent gene pairs in Candida albicans (Arnone \& McAlear, 2011). These genes remain tightly co-regulated even under changing cellular growth status (Arnone et al., 2012; Dai \& Lu, 2008; Grewal et al., 2005). In addition, elevated levels of gene expression, and silencing/repression of expression have also reported for adjacent genes (Grunstein, 1997). This correlation between the expression levels of genes and their relative location to each other can be explained by multiple biochemical, evolutionary, genetic, and technological factors

*E-mail: kirsten.heimann@jcu.edu.au

๑ Acta Facultatis Pharmaceuticae Universitatis Comenianae 
(Bozinovic et al., 2013; Fraser, 2013; Gilad et al., 2006; Hurst et al., 2004; Michalak, 2008; Sproul et al., 2005). For example, it has been theorised that co-expression of adjacent genes can be defined by chromatin domains (Hurst et al., 2004), i.e. unzipping chromatin during gene expression can concurrently facilitate expression of genes from neighbouring opened region (Sproul et al., 2005).

In spite of the potential importance of variation of gene regulation, so far little is known about the effects of selective pressures acting on regulatory patterns. Correlation between gene expression and selective pressure has been observed in model organisms and primates (Gilad et al., 2006). These findings suggest that statistically significant changes in gene expression contribute to phenotypic changes and large morphological differences (Bozinovic et al., 2013). For example in humans, selective pressure in the form of solar radiation is a probable explanation for observed changes in expression levels of genes involved in the UV radiation response, diabetes-related pathways and immune cell proliferation (Fraser, 2013).

The HIS4 (histidinol dehydrogenase) gene is essential for histidine biosynthesis and its transcriptional regulation has been studied extensively in S. cerevisiae. Transcriptional control of HIS4 is carried out by either of two mechanisms: "basal control" is driven by transcriptional factors Bas 1 and Bas2 binding independently to the HIS4 promoter under amino acid-rich condition while "general control" is driven by the transcriptional factor Gcn4p, which is activated under starvation of even a single amino acid and leads to an induction of 40 genes in 12 pathways required for the biosynthesis of amino acids (Lamas-Maceiras et al., 1999; Zaman et al., 1999). Gcn4p binds as a homodimer protein to the consensus sequence $\operatorname{rrTGASTCA}(\mathrm{T}) \mathrm{n}$ and activates the transcription of genes in either direction at a distance of approximately 600 bp. Five such binding sites have been identified in the HIS4 promoter of S. cerevisiae (Lamas-Maceiras et al., 1999). The Gcn $4 p$ gene itself is regulated by the fluctuation of amino acid availability (Zaman et al., 1999). It has also been shown that amino acid starvation can increase HIS4 expression three to four-fold above unstressed levels (Hinnebusch, 2005).

The aim of this study was to assess the effect of amino acid starvation-induced selective pressure on HIS4 on the level of expression of adjacent genes in the recombinant Pichia pastoris. For this purpose; the human interferon gamma $(h I F N Y)$ gene, which has a therapeutic value against wide variety of diseases like cancer, hepatitis and tuberculosis (Miller et al., 2009), was cloned into the pPIC9 vector adjacent to the HIS4 gene. Then it was transformed into the Pichia pastoris GS115 strain, a histidine-deficient mutant. The transformant, containing the HIS4 gene, was cultured under continuous amino acid starvation in modified Yeast Nitrogen Based medium (YNB) void of amino acids, leading to an expression of the HIS4 gene. Finally the expression levels of $h I F N y$ were measured to evaluate the possibility of coregulation of these adjacent genes.

\section{MATERIAL AND METHODS}

\subsection{Cloning and transformation}

Cloning: To generate pPIC9-hIFNy, the coding sequence of $h I F N y$, flanked with EcoRl and Notl, was synthesised by Life Technologies, GeneArt Strings, and modified based on the codon preference in P. pastoris. Subsequently, the fragment was inserted into the PPIC9 vector between the same restriction sites; adjacent to the HIS4 gene, which is essential for biosynthesis of histidine. The optimised sequence encoding $h I F N Y$ and its resultant amino acid sequences are shown in (Fig.1-A).

Transformation \& integration in P. pastoris: The non-linearized plasmid pPIC9-hIFNy was transformed into the GS115 strain of $P$. pastoris by electroporation (Electroporator 2510, Eppendorf) following the protocols for electro-competent cell production and electroporation (Life Technologies). Gene integration occurs at the AOX (GS115) locus by a single crossover between the $A O X$ locus and any of the three $A O X$ regions on the vector: the $5^{\prime} A O X$ promoter, the $A O X$ transcription termination region (TT) or the $3^{\prime} A O X$. This results in the integration of one or more copies of the vector into the genome with the resultant phenotype of $\mathrm{His}^{+} \mathrm{Mut}{ }^{+}$for the transformed P. pastoris (GS115) (Fig.1-B).

Screening for $\mathrm{Mut}^{+}$transformants: Transformant colonies with $\mathrm{HIS4}^{+}$phenotype were selected on Minimal Dextrose (MD) $(1.34 \%$ YNB, 2\% dextrose) agar plates based on complementation of histidine auxotrophy. In order to confirm the methanol utilization (Mut) phenotype of the strain, colonies with $\mathrm{HIS4}^{+}$phenotype were transferred to plates with either MD or Minimal Methanol (MM) (1.34\% YNB, 0.5\% methanol) as the carbon source. This allows to differentiate between Mut $^{5}$ (slow methanol utilisation) and Mut $^{+}$(can utilise methanol effectively as a carbon source) phenotypes, with the latter growing well on MM agar plates, while the former shows insignificant growth. As expected, only the $\mathrm{Mut}^{+}$phenotype was detected based on growth on both agar media after $24 \mathrm{~h}$.

\subsection{Confirmation of integration to genomic DNA by PCR}

In order to determine whether $h I F N \gamma$ was integrated into the $P$. pastoris genome, genomic DNA from colonies with $\mathrm{HIS4}^{+} \mathrm{Mut}^{+}$ phenotype were isolated (Wizard ${ }^{\circledR}$ Genomic DNA Purification Kit, Promega). The integration of $h I F N y$ into the genome of $P$. pastoris was confirmed by $P C R$ using the $a$-Factor sequencing primer as a forward primer 5'-TACTATTGCCAGCATTGCTGC-3' which hybridises within the $5^{\prime}$ end of the a-factor region paired with the $3^{\prime} A O X 1$ sequencing primer as a reverse primer 5'-GCAAATGGCATTCTGACATCC-3' which hybridises with $3^{\prime}$ end of the $A O X 1$ transcription terminator (TT) region (Fig.1). Genomic DNA of untransformed P. pastoris GS115 was used as a negative control. Thirty amplification cycles were 


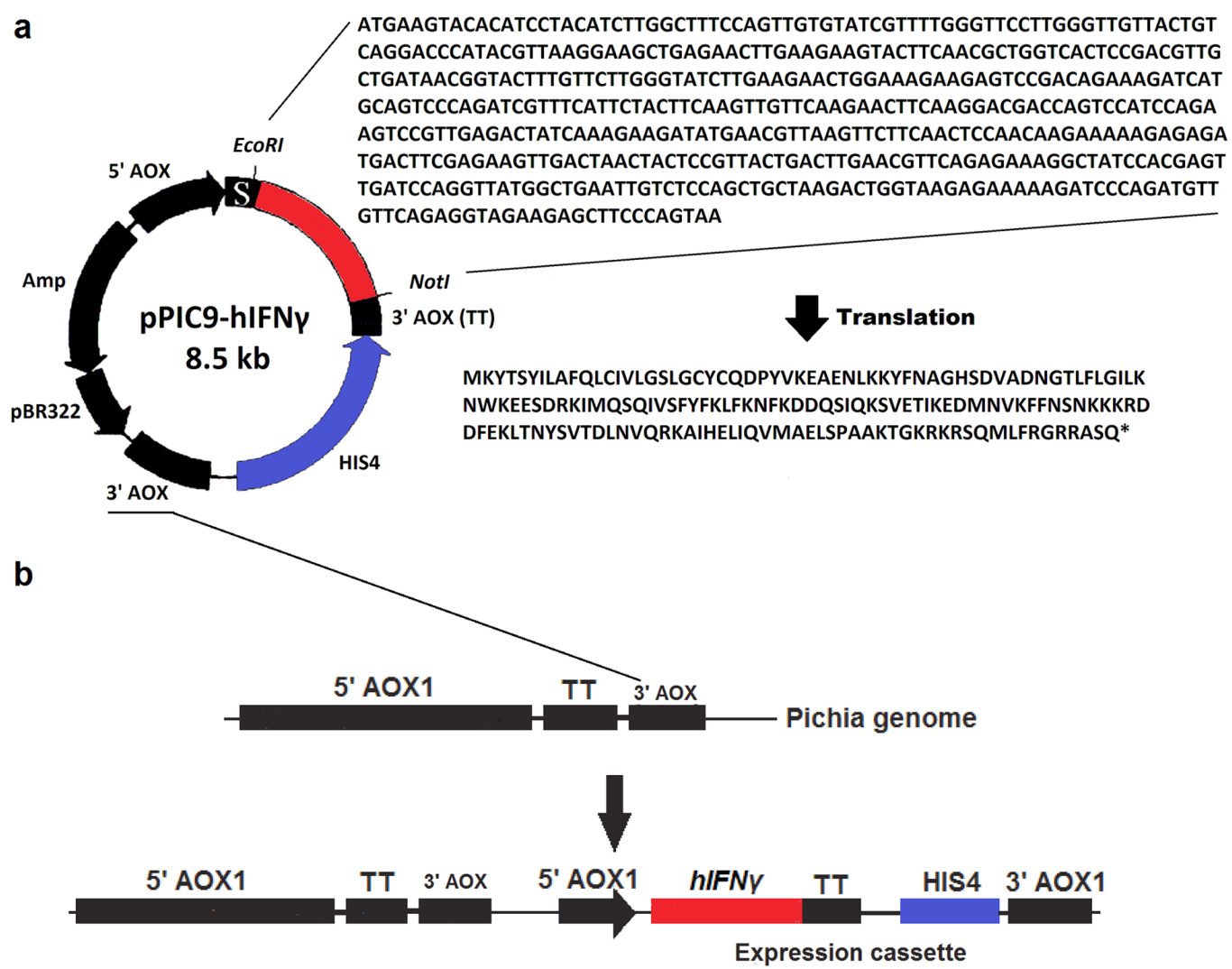

Figure 1. Placement of the two adjacent genes, hIFNY and HIS4, as part of the pPIC9-hIFNY vector (a) and result of the integration of the vector between the $3^{\prime}$ 'AOX into the intact AOX1 locus (Mut ${ }^{+}$) and the gain of promoter 5' AOX1, hIFNy gene, and HIS4 (expression cassette) (b). 5' and AOX1: 5' Alcohol oxidase promotor gene which requires methanol for induction, S: a-factor secretion signal, hIFNY: optimised human interferon gamma gene for P. pastoris, 3' and AOX (TT): Alcohol oxidase transcription terminator, HIS4: Histidinol dehydrogenase gene which is essential for histidine biosynthesis, pBR322: origins from E. coli, Amp: Ampicillin resistance gene

performed at $94^{\circ} \mathrm{C}$ for $30 \mathrm{~s}, 55^{\circ} \mathrm{C}$ for $30 \mathrm{~s}$, and a final extension for 5 min at $72^{\circ} \mathrm{C}$.

Successful integration of the $h I F N Y$ into the P. pastoris genome was demonstrated by the expected $\sim 700$ bp fragment size using agarose (1.5\%) gel electrophoresis which was verified by DNA sequencing at the Australian Genome Research Facility Ltd. (AGRF).

\subsection{Protein expression under amino acid starvation- induced selective pressure on HIS4}

Successfully transformed $P$. pastoris cells were kept under amino acid starvation by cultivation in buffered Minimal Glycerol (BMG) medium (1.34\% YNB without amino acids, 100 $\mathrm{mM}$ potassium phosphate, $\mathrm{pH} 6.0$, and $1 \%$ glycerol). Under these conditions, only successfully transformed cells are able to synthesise histidine and therefore thrive. Explicitly, continuous amino acid starvation was maintained for 10 days; re-inoculating into fresh BMG medium every 2 days (Fig. 2A). A HIS4 $4^{+}$colony was inoculated into $25 \mathrm{~mL}$ of BMG in a $250 \mathrm{~mL}$ baffled flask and incubated at $28^{\circ} \mathrm{C}$ for $48 \mathrm{~h}$ with a shaking speed of $200 \mathrm{rpm}$ until reaching an $\mathrm{OD}_{600} \geq 2$ (log-phase growth) (EnSpire ${ }^{\circledR}$ Multimode Plate Reader, PerkinElmer). Subsequently, the cells were harvested by centrifugation at $3000 \mathrm{~g}$ for $5 \mathrm{~min}$ at room temperature. Cell pellets were resuspended in $50 \mathrm{~mL}$ PBS buffer (0.1 M Phosphate Buffer Saline, $\mathrm{pH}$ 7.4) to remove residual glycerol. Finally cell pellets were resuspended in $50 \mathrm{~mL}$ buffered methanol-complex (BMMY) medium (1\% yeast extract, $2 \%$ peptone, $1.34 \%$ YNB, $100 \mathrm{mM}$ potassium phosphate, $\mathrm{pH} 6.0,0.5 \%$ methanol) to a starting $\mathrm{OD}_{600}=1$ in a $250 \mathrm{~mL}$ baffled flask (Fig.2-B). To induce expression of $h I F N \gamma$, pure methanol was added to a final concentration of $1 \%(\mathrm{v} / \mathrm{v})$ every $24 \mathrm{~h}$. Culture supernatant was obtained by centrifugation at $1500 \mathrm{~g}$ after $72 \mathrm{~h}$ of cultivation to analyse expression of $h I F N \gamma$, cell pellets were used for genomic DNA extraction for qPCR and total RNA extraction for RT-qPCR (Section 2.5).

\subsection{ELISA}

Recombinant $h I F N \gamma$ protein levels in supernatants were quantified using a modified indirect ELISA protocol (Abcam). 


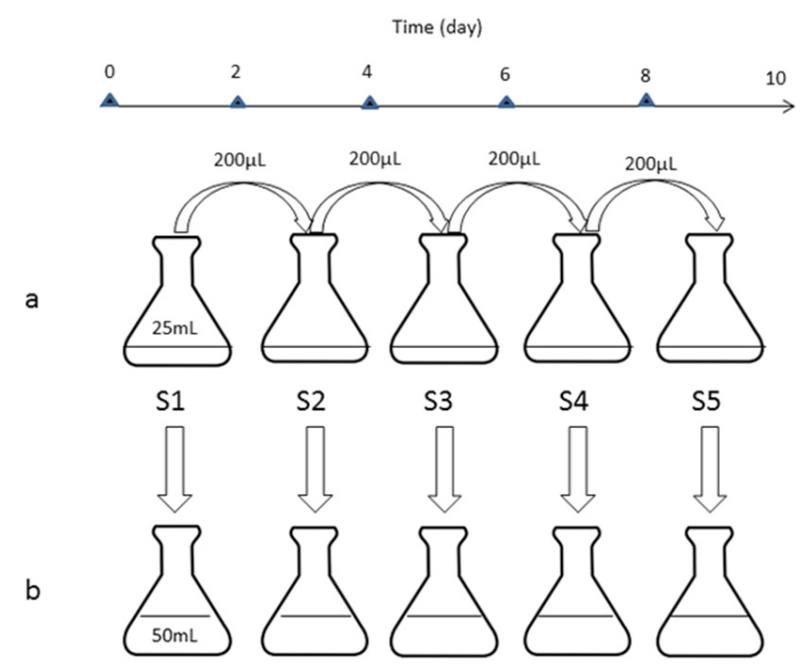

Figure 2. Diagram showing continuous amino acid starvation over 10 days in buffered Minimal Glycerol (BMG) medium (a) and protein expression in buffered methanol-complex (BMMY) medium (b). S: Serial passage
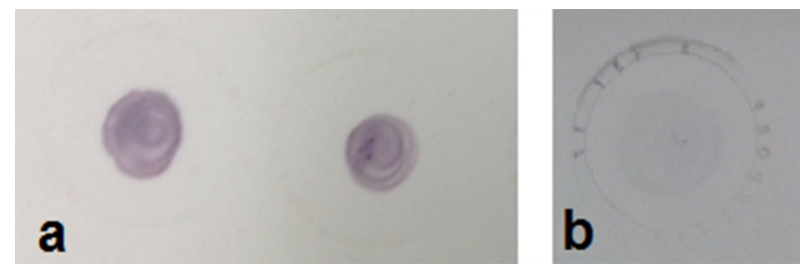

Figure 3. Dot blot showing hIFNy-positive cultivation media of two cultures exposed to amino acid starvation (a) and supernatant of cell culture of untransformed P. pastoris GS115 (negative control) (b)

Replicated sample aliquots $(50 \mu \mathrm{L})$ were added to each well of a polyvinyl chloride micro-titre plate and incubated overnight at $4^{\circ} \mathrm{C}$. Wells were washed three times with $200 \mu \mathrm{L}$ Tris-buffered saline (Tris- $\mathrm{HCl} 20$ mM, NaCl $150 \mathrm{mM}$, $\mathrm{pH}$ 7.5). Protein-binding sites were blocked by adding $200 \mu \mathrm{L}$ blocking buffer (5\% Bovine Serum Albumin (BSA) in TBS) per well, followed by incubation for $1 \mathrm{~h}$ at $37^{\circ} \mathrm{C}$ in a shaking incubator chamber $\left(\mathrm{HO}_{3} 5^{\mathrm{TM}}\right.$ Hybridisation Oven, Ratek), followed by washing twice with TBS. $100 \mu \mathrm{L}$ of diluted $\left(0.5 \mu \mathrm{g} \cdot \mathrm{mL}^{-1}\right)$ primary antibody (polyclonal rabbit-anti-hIFN , Abcam cat no. ab9657) was added to each well and incubated for $1 \mathrm{~h}$ at $37^{\circ} \mathrm{C}$ in a shaking incubator chamber. Plates were washed four times with TBS. $100 \mu \mathrm{L}$ of conjugated secondary antibody (polyclonal goat anti-rabbit, Abcam cat no. ab98505) diluted 1/1000 in blocking buffer was added to each well and incubated for $1 \mathrm{~h}$ at $37^{\circ} \mathrm{C}$ in a shaking incubator chamber. After washing four times with TBS, $50 \mu \mathrm{L}$ of Alkaline Phosphatase Yellow (pNPP) Liquid Substrate (P7998 SIGMA) was added per well. Absorbance at $405 \mathrm{~nm}$ was recorded after $30 \mathrm{~min}$ on a spectrophotometer (EnSpire ${ }^{\circledast}$ Multimode Plate Reader,
PerkinElmer). Supernatant of cell culture of untransformed P. pastoris GS115was used as negative controls.

A standard curve $0,1.25,2.5,5,10 \mu \mathrm{g} . \mathrm{L}^{-1}\left(R^{2}=0.993\right.$ and $y=12.169 x-1.1321$ ) was prepared by serial dilution of the recombinant $h I F N \gamma$ (Abcam cat no. ab51240).

\subsection{Immuno-blotting}

Immunoblotting (dot blot) was performed to qualitatively detect the presence of hIFNY in the medium supernatant, following standard procedures described by Abcam. In brief, a nitrocellulose membrane (pore size $0.2 \mu \mathrm{m}$ N7892 SIGMA) was gridded and $2 \mu \mathrm{L}$ of samples were spotted onto the nitrocellulose membrane at the centre of each grid square. The membrane was left to dry for $30 \mathrm{~min}$. Unspecific binding sites were blocked with 1\% BSA in TBS-T (Tris buffered saline-TWEEN 20 0.05\%) for $30 \mathrm{~min}$ at room temperature on a rocking shaker (VSR-50 ${ }^{\circledR}$ Laboratory Platform Rocker). The membrane was then incubated with the primary antibody (polyclonal rabbit anti-hIFNY, Abcam cat no. ab9657) (0.1 $\left.\mu \mathrm{g} \cdot \mathrm{mL}^{-1}\right)$ dissolved in TBS-T over night at room temperature. The membrane was washed for 5 min three times with TBS-T. Thereafter, the membrane was incubated for $2 \mathrm{~h}$ at room temperature with the secondary antibody (polyclonal goatanti-rabbit, Abcam cat no. ab98505), conjugated to alkaline phosphatase. Finally, the membrane was washed twice for 5 min with TBS-T and incubated with SIGMA FAST" BCIP/ NBT (5-bromo-4-chloro-3-indolyl phosphate/nitro blue tetrazolium) dissolved in $10 \mathrm{~mL}$ deionised water, and left until colour developed (Fig. 3). Supernatant of cell culture of untransformed $P$. pastoris GS115 was used as negative controls.

\section{6. qPCR, RNA EXTRACTION \& RT-qPCR}

QuantiTect SYBR Green PCR Kit (Qiagen cat no. 204141) was used for both real time PCR (qPCR; assessing gene copy number) and two-step reverse transcription-PCR (RT-qPCR; assessing transcription level of the RNA) using primers as per Table 1. A set of primers was designed to amplify $168 \mathrm{bp}$ of the hIFNY sequence (Section 2.1).

Genomic DNA of each serial passage was extracted at the end of the experiment, and 50 ng of DNA (NanoDrop ${ }^{\circledR}$, ND1000 Spectrophotometer) for each serial passage was used in qPCR. Total RNA was extracted using the PureLink ${ }^{\circledast}$ RNA Mini Kit (Life Technologies cat no. 12183018A), followed by DNase treatment and reverse transcription to CDNA using the QuantiTect Reverse Transcription Kit (Qiagen cat no. 205310). For each serial passage, 500 ng of cDNA was quantified by NanoDrop based on the optical absorbance at $\mathrm{OD}_{260^{\circ}}$. Two replicated RT-qPCRs were performed using the same primer set as for qPCR (Table 1). Genomic DNA and total RNA of untransformed P. pastoris GS115 was used as negative controls for $\mathrm{QPCR}$ and RT-qPCR, respectively. 
Table 1. Primer design for $q P C R / R T-q P C R$.

\begin{tabular}{|c|c|c|c|c|}
\cline { 2 - 5 } \multicolumn{1}{c|}{} & Sequence & Length [bp] & T $_{\mathbf{m}}$ & GC\% \\
\hline Forward primer & 5'ACTTCAACGCTGGTCACTC 3' & 19 & 57.71 & 52.63 \\
\hline Reverse primer & 5'CGGACTTCTGGATGGACTG 3' & 19 & 57.25 & 57.89 \\
\hline
\end{tabular}

Standard curves for qPCR were prepared with purified DNA amplicons (section 2.2) [699 bp amplicon containing 501 bp $h I F N Y$ plus secretion signal and parts of the AOX gene promoter and transcription terminator]. Dilution series of DNA amplicons according to mass concentration ( $\mathrm{ng} / \mathrm{per}$ total volume of reaction) were used to make standard curves with, $10^{-3}, 10^{-4}, 10^{-5}, 10^{-6}$ and 0 ng DNA $\left(R^{2}=0.998\right.$, Overall efficiency $=101.1 \%$ and $\left.y=11.149 \mathrm{e}^{-0.699 x}\right)$.

For calculation of approximate gene copy number the following equation was used, based on the fact that $699 \mathrm{bp}$ dsDNA amplicon weighs $\sim 75.33^{*} 10^{-10} \mathrm{ng}$.

Gene copy number = Initial concentration of detected DNA amplicon (ng) / $75.33^{*} 10^{-10} \mathrm{ng}$.

Each $50 \mu \mathrm{L}$ reaction contained $25 \mu \mathrm{L}$ (2x) QuantiTec SYBR GreenPCR Master Mix, $10 \mu \mathrm{M}$ forward and reverse primers with final concentration of $0.3 \mu \mathrm{M}$ ( $5 \mu \mathrm{L}$ each), $10 \mu \mathrm{L}$ sample (genomic DNA or CDNA) and $5 \mu \mathrm{L}$ RNase-free water. qPCR reactions were run on a Peltier Thermal Cycler-200 (BioRad) under the following conditions: PCR initial activation step $95^{\circ} \mathrm{C}$ for $15 \mathrm{~min}$, followed by 45 cycles of denaturation at $94^{\circ} \mathrm{C}$ for $15 \mathrm{~s}$ and annealing at $57^{\circ} \mathrm{C}$ for $30 \mathrm{~s}$, and extension at $72^{\circ} \mathrm{C}$ for $30 \mathrm{~s}$.

\subsection{Statistical analysis}

Data on hIFNy protein secretion levels and C(t) of RT-qPCR were statistically analysed via one-way ANOVA using Microsoft Office Excel, Data Analysis. Homogeneity of variances was confirmed using Levene's Test. Critical value was set to $(a=0.05)$ and results were deemed statistically significant at $p \leq 0.05$. If statistical significance was detected, Tukey-Kramer HSD post-hoc tests were performed to identify samples significantly different to each other.

\section{RESULTS}

\subsection{Transformation and confirmation of integration}

Six clones were retained and their phenotypic $\mathrm{HIS4}^{+}$status was confirmed on Minimal Dextrose and $\mathrm{Mut}^{+}$status on Minimal Methanol agar plates. The successful integration of the hIFNY was confirmed by PCR using $5^{\prime}$ and $3^{\prime}$ primers of the $A O X 1$ and a-factor partial sequence, respectively (Section 2.2). Agarose gel electrophoresis of $\mathrm{HIS4}^{+} \mathrm{Mut} \mathrm{t}^{+}$transformants confirmed PCR products between 500-1000 bp according to a DNA ladder (EasyLadder I, Bioline), while negative controls

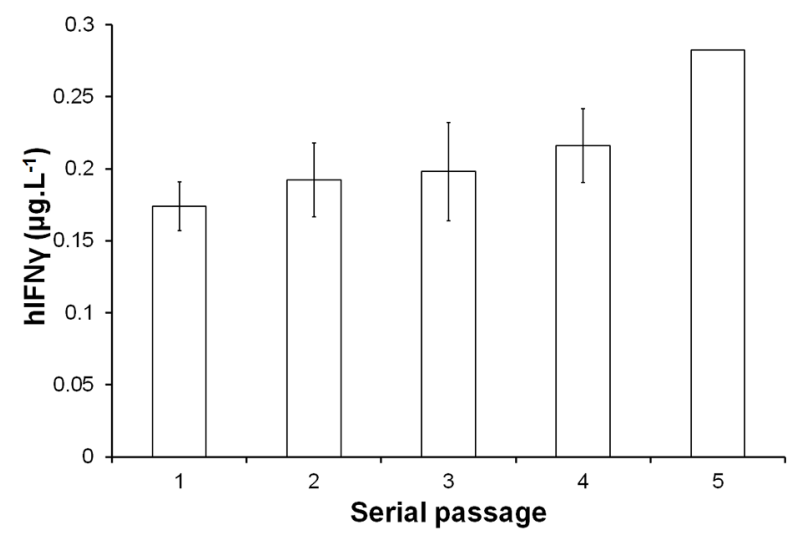

Figure 4. Amino acid starvation-induced levels of secreted hIFNy over 5 serial passages of $P$. pastoris GS115 transformed with hIFNY and HIS4 (Mean $\pm S D, n=2$ )

(untransformed P. pastoris) showed no band. Results of DNA sequencing at the Australian Genome Research Facility Ltd. (AGRF) confirmed that amplicons sequences match the optimised $h I F N \gamma$ (Section 2.1).

\subsection{Protein expression under amino acid starvation- induced selective pressure on HIS4}

The strain (C6) yielded the strongest agarose gel signal and was used for protein expression and secretion studies (Section 2.3). The amount of secreted hIFNY was assessed $72 \mathrm{~h}$ after induction in BMMY medium by immunoblots (Fig. 3) and ELISA, with the latter detecting secreted yields of 0.18 to $0.28 \mu \mathrm{g} . \mathrm{L}^{-1}$ of $h I F N \gamma$ (Fig. 4). Culture supernatants of the untransformed $P$. pastoris were used as negative controls. Immunodot blots using culture supernatant of the untransformed P. pastoris yielded no positive signal (Fig. 3), further demonstrating that the construct, pPIC9-hIFNy, was successfully expressed in P. pastoris.

Levene's test for determining homogeneity of variances validated the assumption of equal variances $\left(p=4.95^{*} 10^{-75}\right)$. A one-way ANOVA showed that there was a significant difference in levels of $h I F N \gamma$ secretion between one or more pairs of serial passages (Table 2). A significant difference between serial passages 1 and 5 was detected (TukeyKramer HSD test: $p=0.029$ ), while serial passages 2-4 were not significantly different to either serial passage 1 or serial passage 5 ( $p>0.05)$. 
Table 2. Summary of one-way ANOVA results for 5 serial passages of transformed P. pastoris producing hIFNy

\begin{tabular}{|c|c|c|c|c|c|c|}
\hline Source of Variation & SS & df & MS & F & F crit & P-value \\
\hline Between Groups & 0.015841 & 4 & 0.00396 & 6.961538 & 5.192168 & 0.028203 \\
\hline Within Groups & 0.002844 & 5 & 0.000569 & & & \\
\hline Total & 0.018685 & 9 & & & & \\
\hline
\end{tabular}

Table 3. Approximate hIFNY gene copy number and hIFNY DNA amplicon concentration [ng] of serial passages 1, 3 and 5 of hIFNY $-H I S 4^{+}$Mut $^{+}$P. pastoris transformants under amino acid starvation

\begin{tabular}{|c|c|c|c|}
\hline Content & $\mathbf{C}(\mathbf{t})$ & Initial Concentration of $\boldsymbol{h I F N} \boldsymbol{\gamma}$ DNA amplicons (ng) & Approx. gene copy number \\
\hline Serial passage 1 & 13.93 & $65.84^{*} 10^{-5}$ & $\sim 87.34^{*} 10^{3}$ \\
\hline Serial passage 3 & 13.79 & $72.61^{*} 10^{-5}$ & $\sim 96.37^{*} 10^{3}$ \\
\hline Serial passage 5 & 13.97 & $64.02^{*} 10^{-5}$ & $\sim 85.96^{*} 10^{3}$ \\
\hline
\end{tabular}

Table 4. $C(t)$ values of $R T-q P C R$ for quantification of hIFNY RNA and calculated initial concentration of the CDNA amplicons (Mean $\pm S D, n=2$ )

\begin{tabular}{|c|c|c|}
\hline Content & $\mathbf{C}(\mathbf{t})$ & $\begin{array}{c}\text { Initial Concentration of } \\
\text { CDNA amplicons (ng) }\end{array}$ \\
\hline $\begin{array}{c}\text { Serial } \\
\text { passage 1 }\end{array}$ & $24.79 \pm 0.035$ & $3.34^{*} 10^{-07} \pm 0.08^{*} 10^{-07}$ \\
\hline $\begin{array}{c}\text { Serial } \\
\text { passage 3 }\end{array}$ & $25.02 \pm 0.162$ & $2.89^{*} 10^{-07} \pm 0.33^{*} 10^{-07}$ \\
\hline $\begin{array}{c}\text { Serial } \\
\text { passage 5 }\end{array}$ & $23.28 \pm 0.028$ & $9.55^{*} 10^{-07} \pm 0.19^{*} 10^{-07}$ \\
\hline
\end{tabular}

\subsection{Gene quantification and gene copy number analysis}

The results of the $\mathrm{qPCR}$ for determining concentration of target gene showed consistent and similar amounts of amplified the 168 bp amplicon, suggesting equal hIFNY gene numbers across serial passages (Table 3 ).

\subsection{Transcriptional analysis of hIFN $\gamma$ RNA}

Levene's test for determining homogeneity of variances validated the assumption of equal variances $\left(p=1.3^{*} 10^{-41}\right)$. A one-way ANOVA on $C(t)$ values showed that there was a significant difference between one or more pairs of serial passages $(p=0.0007)$. A Tukey-Kramer HSD test revealed no significant differences between serial passages 1 and 3 $(p>0.01)$, but a significant difference between serial passages 1 and $5(p<0.01)$ and serial passages 3 and $5(p<0.001)$. These results conformed to the protein expression/secretion results obtained by ELISA (section 3.2).

\section{DISCUSSION}

Studies in model organisms propose that the expression levels of most genes change and evolve under stabilising selective pressure which has been proposed to be the dominant mode of evolutionary changes in gene expression (Gilad et al., 2006). Gene expression in yeast has also been shown to change in response to environmental stress; for example, the expression of a significant number of genes (1372) was altered distinctively when $S$. cerevisiae was cultivated for either five or twenty five generations under microgravity to compared controls cultured under identical conditions in normal gravity (Sheehan et al., 2007). Therefore, as a driving force of evolution, the outcomes of selective pressures are generally well documented, while the linkage to direct genetic effects are less understood.

Much of the work on regulatory networks has focused on the yeast $S$. cerevisiae, for which data are most copious (Babu et al., 2004). To the best of our knowledge, the effects of amino acid starvation-induced selective pressure on HIS4 and the transcriptional co-regulation of recombinant adjacent genes in P. pastoris has not been documented to date. As predicted in our study, amino acid starvation-induced selective pressure on HIS4 increased expression of the adjacent hIFNY gene by $\sim 55 \%$, suggesting co-regulation, as increased secretion levels were positively correlated with RNA transcription levels. Investigation of gene copy number of hIFNY in every other serial passage showed no variation (Table 3), suggesting that increased level of protein expression and RNA transcription is not due to "gene duplication" making transcriptional coregulation between hIFNY and the adjacent HIS4 gene highly likely.

At least three mechanisms have been proposed to explain adjacent gene co-regulation: 
1. Localised chromatin modification; where there is a correlation between histone $\mathrm{H} 4$ acetylation domains and genome-wide histone H3K14 acetylation which correlate with transcriptionally co-expressed genes in budding yeast (Deng et al., 2010). When a gene is being transcribed, the localised chromatin is forming a more open permissive transcriptional state (Sproul et al., 2005), which can affect the transcription of genes in adjacency (Ebisuya et al., 2008).

2. Local DNA sequence looping; which has been observed between genes on the same and different chromosomes in yeast (Duan et al., 2010), where adjacent genes can be silenced via a localised loop of DNA sequences when the promoter of the adjacent gene is in physical contact with silencing factors (Valenzuela et al., 2008).

3. Adjacent gene co-regulation through sub-nuclear compartmentalisation; where transcriptionally active sets of genes are lodged at the nucleolar periphery upon activation (Berger et al., 2008). Active genes have been seen to associate with 'transcription factories', which are the spot of nascent RNA production and associated transcription factors (Osborne et al., 2004). As a result, if one gene gains entry to an active sub-nuclear compartment, the adjacent gene could hypothetically follow the same regulatory process (Arnone et al., 2012).

In the study here, while gene co-regulation through DNA looping are possible scenarios for other non-investigated genes, expression of HIS4 would have been activated by amino acid starvation (as shown by (Hinnebusch, 2005)), and, since increased transcription (mRNA) and expression/ secretion was also improved, co-regulation of these two genes would need to be achieved through either mechanism 1 or 3. It is much harder to differentiate between the potential roles of localised chromatin modifications and sub-nuclear compartmentalisation in the co-regulation of HIS4 and hIFNy, because these two mechanisms are not mutually exclusive in operation. There is some evidence supporting "localised chromatin modifications" playing 'a' greater role in gene co-regulation in eukaryotes e.g. yeast (Babu et al., 2004). Latest evidence also suggests that the environment can stably affect the establishment of the epigenome which is referred to as "transgenerational epigenetic inheritance" (Daxinger \& Whitelaw, 2010). The role of localised chromatin modifications as an epigenomic co-regulatory mechanism would require investigating the stable inheritance of HIS4 and hIFNY expression when the selective pressure of amino acid starvation is removed. This should be ideally conducted simultaneously with experiments aiming to identify transcriptional localisation of HIS4 and hIFN $\gamma$ within the nucleus to examine the potential for a contribution of nucleolar transcriptional factories.

It could be equally argued that gene co-expression might be regulated by simple regulatory networks, which do not necessarily require any of the above regulatory mechanisms. For example, it was shown that external conditions e.g. stress induced topologically simple regulatory networks, characterised by involving a limited number of steps and transcription factors in yeast (Babu et al., 2004). In relevance to the study here, amino acid starvation activated the transcription factor Gcn4p, resulting in transcriptional induction of almost all genes involved in amino acid biosynthesis (Hinnebusch, 2005), including HIS4. Additionally, a wide array of genes unrelated to amino acid biosynthesis, i.e. close to one tenth of the yeast genome was activated (Natarajan et al., 2001), which designates a role for Gcn4p as a "master regulator" for gene expression (Hinnebusch \& Natarajan, 2002). Thus involvement of Gcn4p in regulation of both HIS4 and hIFNY can be hypothesised as a probable scenario explaining the increased level of $h I F N \gamma$ under amino acid starvation.

\section{CONCLUSION}

This study showed that the adjacent localisation of hIFNY and HIS4 genes result in co-regulation of hIFNY expression and secretion, a first step for potential improvement of hIFNY yields using this expression system. Additionally, the recombinant system developed should lend itself for detailed studies regarding the underpinning nature of the regulatory mechanism.

\section{ACKNOWLEDGEMENTS}

$\mathrm{KH}$ acknowledges project support by the Advanced Manufacturing Cooperative Research Centre (AMCRC), funded through the Australian Government's Cooperative Research Centre Scheme, grant number 2.3.4. The funders had no role in study design, data collection and analysis or preparation of the manuscript and have provided permission to publish. This research is part of the MBD Energy Research and Development program for Biological Remediation of Methane from Underground Coal Mine Ventilation Air. Ali Razaghi was supported by an AMCRC PhD scholarship. 
[1] Arnone J, McAlear M. Adjacent gene pairing plays a role in the coordinated expression of ribosome biogenesis genes MPP10 and YJR003C in Saccharomyces cerevisiae. Eukaryot Cell. 2011;10:43-53.

[2] Arnone JT, Robbins-Pianka A, Arace JR, Kass-Gergi S, McAlear MA. The adjacent positioning of co-regulated gene pairs is widely conserved across eukaryotes. BMC Genomics. 2012;13:546-561.

[3] Babu M, Luscombe N, Aravind L, Gerstein M, Teichmann S. Structure and evolution of transcriptional regulatory networks. Curr Opin Struct Biol. 2004;14:283-291.

[4] Berger A, Cabal G, Fabre E et al. High-resolution statistical mapping reveals gene territories in live yeast. Nature Methods. 2008;5:1031-1037.

[5] Boutanaev AM, Kalmykova Al, Shevelyov YY, Nurminsky DI. Large clusters of co-expressed genes in the Drosophila genome. Nature. 2002;420:666-669.

[6] Bozinovic G, Sit TL, Di Giulio R, Wills LF, Oleksiak MF. Genomic and physiological responses to strong selective pressure during late organogenesis: few gene expression changes found despite striking morphological differences. BMC Genomics. 2013;14:779_ 794.

[7] Dai M, Lu H. Crosstalk between c-Myc and ribosome in ribosomal biogenesis and cancer. J Cell Biochem. 2008;105:670-677.

[8] Daxinger L, Whitelaw E.Transgenerational epigenetic inheritance: More questions than answers. Genome Res. 2010;20:1623-1628.

[9] Deng Y, Dai X, Xiang Q et al. Genome-wide analysis of the effect of histone modifications on the coexpression of neighboring genes in Saccharomyces cerevisiae. BMC Genomics. 2010;11:550-566.

[10] Duan Z, Andronescu M, Schutz K et al. A three-dimensional model of the yeast genome. Nature. 2010;465:363-367.

[11] Ebisuya M, Yamamoto T, Nakajima M, Nishida E. Ripples from neighbouring transcription. Nature Cell Biol. 2008;10:1106-1113.

[12] Fraser HB. Gene expression drives local adaptation in humans. Genome Res. 2013;23:1089-1096.

[13] GiladY, Oshlack A, Rifkin SA. Natural selection on gene expression. Trends Genet. 2006;22:456-461.

[14] Grewal S, Li L, Orian A, Eisenman R, Edgar B. Myc-dependent regulation of ribosomal RNA synthesis during Drosophila development. Nature Cell Biol. 2005;7:295-302.

[15] Grunstein M. Molecular model for telomeric heterochromatin in yeast. Curr Opin Cell Biol. 1997;9:383-387.

[16] Hinnebusch AG. Translational regulation of GCN4 and the general amino acid control of yeast. Annu Rev Microbiol. 2005;59:407450.

[17] Hinnebusch AG, Natarajan K. Gcn4p, a master regulator of gene expression, is controlled at multiple levels by diverse signals of starvation and stress. Eukaryotic Cell. 2002;1:22-32.
[18] Hurst LD, Pal C, Lercher MJ. The evolutionary dynamics of eukaryotic gene order. Nature Rev Genet. 2004;5:299-310.

[19] Kruglyak S, Tang H. Regulation of adjacent yeast genes. Trends Genet. 2000;16:109-111.

[20] Lamas-Maceiras M, Cerdán ME, Freire-Picos MA. Kluyveromyces lactis HIS4 transcriptional regulation: similarities and differences to Saccharomyces cerevisiae HIS4 gene. FEBS Lett. 1999;458:7276.

[21] Lercher MJ, Blumenthal T, Hurst LD. Coexpression of neighboring genes in Caenorhabditis elegans is mostly due to operons and duplicate genes. Genome Res. 2003;13:238-243.

[22] Michalak P. Coexpression, coregulation, and cofunctionality of neighboring genes in eukaryotic genomes. Genomics. 2008;91:243-248.

[23] Miller CH, Maher SG, Young HA. Clinical use of interferon-gamma. Annals of the New York Academy of Sciences. 2009;1182:69-79.

[24] Natarajan K, Meyer MR, Jackson BM et al. Transcriptional profiling shows that Gcn4p is a master regulator of gene expression during amino acid starvation in yeast. Mol Cell Biol. 2001;21:4347-4368.

[25] Osborne C, Chakalova L, Brown K et al. Active genes dynamically colocalize to shared sites of ongoing transcription. Nature Genet. 2004;36:1065-1071

[26] Purmann A, Toedling J, Schueler M et al. Genomic organization of transcriptomes in mammals: Coregulation and cofunctionality. Genomics. 2007;89:580-587.

[27] Sheehan KB, Mclnnerney K, Purevdorj-Gage B, Altenburg SD, Hyman LE. Yeast genomic expression patterns in response to low-shear modeled microgravity. BMC Genomics. 2007;8:3-15.

[28] Sproul D, Gilbert N, Bickmore WA. The role of chromatin structure in regulating the expression of clustered genes. Nature Rev Genet. 2005;6:775-781.

[29] Valenzuela L, Dhillon N, Dubey R, Gartenberg M, Kamakaka R. Long-range communication between the silencers of HMR. Mol Cell Biol. 2008;28:1924-1935.

[30] Wade C, Umbarger M, McAlear M. The budding yeast rRNA and ribosome biosynthesis (RRB) regulon contains over 200 genes. Yeast. 2006;23:293-306.

[31] Wei W, Pelechano V, Jarvelin A, Steinmetz L. Functional consequences of bidirectional promoters. Trends Genet. 2011;27:267-276.

[32] Williams EJ, Bowles DJ. Coexpression of neighboring genes in the genome of Arabidopsis thaliana. Genome Res. 2004;14:10601067.

[33] Zaman Z, Bowman SB, Kornfeld GD, Brown AJ, Dawes IW. Transcription factor GCN4 for control of amino acid biosynthesis also regulates the expression of the gene for lipoamide dehydrogenase. Biochem J. 1999;340:855-862. 